\title{
Linear Switching State Space (LS3) Model for Task Scheduling: An Analytical Approach
}

\author{
H. Tabatabaee ${ }^{1}$, M.-R.Akbarzadeh-T ${ }^{2}$ and N. Pariz ${ }^{3}$ \\ ${ }^{1}$ Department of Computer Engineering, Islamic Azad University, Quchan Branch, Quchan, Iran \\ ${ }^{2}$ Center of Excellence on Soft Computing and Intelligent Information Processing, \\ ${ }^{23}$ Dept. of Electrical Engineering, Ferdowsi University of Mashhad, Mashhad, Iran \\ E-mail: ' hamid.tabatabaee@gmail.com
}

\begin{abstract}
Task Scheduling (TS) poses a challenging problem in distributed systems such as multiprocessor systems, flow-shop scheduling, and project management problems in which there are multiple tasks and processors (resources), and the problem is to efficiently assign tasks to processors. The importance of this problem includes several aspects such as heterogeneity of processors, computational complexity of reaching a solution as well as theoretical performance analysis. In this paper, control theory is used to construct a modeling paradigm. The approach which is basically a switching state space model opens a possibility of using the extensive theoretical developments that have taken place in this field within the past several decades. In this study, first we show the role of nonlinear state space equations in modeling the standard TS, a suitable transformation is then devised to convert this model to linear switching state space (LS3) equations with nonlinear constraints, and then the most important aspects of a control-based system - the stability, controllability, observability and stabilizability - for the proposed method is analytically proven. Finally we inspected the robustness of the proposed model in a similar way at the presence of the changes in processing power and link failure.
\end{abstract}

Keywords: Task Scheduling, Distributed Systems, Linear Switching State Space Model, Stabilizability, Controllability, Observability.

\section{INTRODUCTION}

Various aspects of task scheduling (TS) have been addressed in the previous works by present. This problem has many real world applications such as management of projects, human resources, financial resources as well as distributed computing systems. In general, a multi-processor system must be both fast as well as it must utilize its available resources efficiently to complete the requested tasks while it may face various challenges such as in processor heterogeneity, interdependent tasks, etc. Finally, inappropriate scheduling of tasks can fail to exploit the true potential of a distributed system and can offset the gains from parallelization due to excessive communication overhead or under-utilization of processing resources [1].

The task scheduling techniques can be divided into two main classes: static and time-varying. It should be mentioned that the word 'dynamic' has dual meanings in the computer/AI and system engineering. Hence, the word 'time varying' is used for 'dynamic' environments here to distinguish the difference in meaning with dynamic systems, i.e. systems that have memory. In static scheduling, information regarding tasks execution times and resources (nodes) are assumed to be known beforehand. Whereas, dynamic scheduling is based on the task assignment during their executions, taking into account overloaded and under-loaded nodes, with the assumption that if the load among all nodes is balanced; the overall execution time of the application would be minimized. Hence, according to either centralized or distributed information, a decision has to be made whether or not a task should be transferred and to which node it should be transferred [2]. 
Until now, many researchers have solved this problem by heuristic and random search methods, but they often consider certain constraints that lead to inapplicability of their methods in the real world. Furthermore, their performance evaluation is often only numeric and lack a modeling strategy and also a proof for system hypotheses. Hence, making a general model for task scheduling problems is essential that would be amenable to theoretical and rigorous analysis.

In this paper, we present a new modeling paradigm based on switching systems theory for theoretical modeling and analysis. This new paradigm, because of its rich mathematical framework, provides rigorous tools of analysis to yield concrete results in the field of task scheduling. It is assumed that tasks are sufficiently decomposed to their integral part such that there is no parallelism within a task and all instructions are executed sequentially. Furthermore, we consider a distributed computing system as a well-known sample for task scheduling problem. In this study, first we show how the standard TS can be modeled using nonlinear state space equations and then mapped to a linear model, while the stability, controllability, observability and stabilizability of task scheduling problem is analytically proven. Also, the robustness of the proposed model is considered in the presence of the changes in processing power and link failure.

The major contributions of this work can be stated as follows [2]:

- The task scheduling problem is modeled by linear switching state space equations. This new model converts the problem of task scheduling to choose among several switches. Hence it simplifies the controller (scheduler) design process.

- The above mapping is a general modeling framework, not just an algorithm, to solve the scheduling problem. In other words, the modeling strategy can be adapted to other variations of the scheduling problem such as job shop and work flow scheduling as well as project management problems.

- The model is capable of supporting multiple constraints in a real environment such as considering the small time steps as well as the optimal routing between processing nodes.
This paper is organized as follows. In Section 2, the fundamentals of switching systems paradigm are stated. In Section 3, some other similar works in the field of task scheduling are reviewed. Section 4 defines the problem specifically and also the proposed method is discussed in details. We study some of the characteristics of the proposed approach from the viewpoint of control theory in Section 5. Finally, Section 6 concludes the proposed method

\section{PRELIMINARIES}

In this section, some basic definitions and axioms that are used in this paper are reviewed from control theory. As three of the most important paradigms, here the concepts of controllability, observability and stabilizability are discussed.

\subsection{CONTROLLABILITY}

The controllability of linear systems is completely defined by the rank of controllability matrix [3]. Controllability has close relevance to pole assignment, structural decomposition and quadratic optimal design.

Definition 1: (State Controllability) A nonzero state $\mathrm{x} \in \mathrm{R}^{\mathrm{n}}$ for $\mathrm{x}\left(\mathrm{t}_{0}\right)=\mathrm{x}_{0}$ is controllable if there exists a time constant $t_{\mathrm{f}}>\mathrm{t}_{0}$, an input $\mathrm{u}$ and a switching path $\sigma:\left[\mathrm{t}_{0}, \mathrm{t}_{\mathrm{f}}\right]$ a $M$ such that $\mathrm{x}\left(\mathrm{t}_{\mathrm{f}}\right)=0$.

Definition 2: (System Controllability) System 1 is set to be completely controllable if any nonzero state of the system 1 is controllable.

Definition 3: (Invariant subspace) For a matrix $\mathrm{A} \in \mathrm{R}^{\mathrm{n} \times \mathrm{n}}$ and a linear subspace $\mathrm{W} \subset \mathrm{R}^{\mathrm{n}}$, invariant subspace is defined as below:

$$
\langle A \mid W\rangle \stackrel{\text { def }}{=} W+A W+\mathrm{L}+A^{n-1} W .
$$

In which $\langle A \mid W\rangle=\langle A \mid R(W)\rangle$ and $R(W)$ is the range of the matrix $W$. For the linear switched system 1, invariant subspace is defined as [4]:

$$
\begin{aligned}
& W_{1}=\sum_{i=1}^{N}\left\langle A_{i} \mid B_{i}\right\rangle \\
& W_{2}=\sum_{i=1}^{N}\left\langle A_{i} \mid W_{1}\right\rangle
\end{aligned}
$$

M

$$
W_{n}=\sum_{i=1}^{N}\left\langle A_{i} \mid W_{n-1}\right\rangle
$$


Thus, necessary and sufficient condition is introduced as:

Axiom 1: (Necessary condition) [5] if the linear switched system is controllable then $W_{n}=R^{n}$

Axiom 2: (Sufficient condition) [5] the linear switched system is controllable if $W_{1}=R^{n}$

Definition 4: (Controllability for a special path) $A$ nonzero state $\mathrm{x} \in \mathrm{R}^{\mathrm{n}}$ for $\mathrm{x}\left(\mathrm{t}_{0}\right)=\mathrm{x}_{0}$ via path $\sigma: R$ a $M$ is controllable if there exist a time constant $t_{f}>t_{0}$ and an input signal $u:\left[t_{0}, t_{f}\right]$ a $\quad R^{p}$ such that $x\left(t_{f}\right)=0$.

\subsection{OBSERVABILITY}

Observability is also one of the important topics of control theory for which we have following definitions.

Definition 4: (state observability) $[6,7] \quad$ A nonzero state $\mathrm{x} \in \mathrm{R}^{\mathrm{n}}$ for $\mathrm{x}\left(\mathrm{t}_{0}\right)=\mathrm{x}_{0}$ is observable at time $t_{0}$, if there exist a time $t_{1}>t_{0}$ and a switching law $\sigma(\mathrm{t})$ defined on $\left[t_{0}, t_{1}\right)$ such that $x_{0}$ can be uniquely determined from the input $u_{\sigma}(t)$, switching law $\sigma(\mathrm{t})$ and output $\mathrm{y}(\mathrm{t}), \mathrm{t} \in[\mathrm{t} 0, \mathrm{t} 1]$.

The system 1 is observable if it is observable in all time instants t. since observability and controllability are dual of each other, so invariant subspace for observability defined as [8]:

$$
\begin{aligned}
& O_{1}=\sum_{i=1}^{N}\left\langle A_{i}^{T} \mid C_{i}^{T}\right\rangle \\
& O_{2}=\sum_{i=1}^{N}\left\langle A_{i}^{T} \mid O_{1}\right\rangle \\
& \mathrm{M} \\
& O_{n}=\sum_{i=1}^{N}\left\langle A_{i}^{T} \mid O_{n-1}\right\rangle .
\end{aligned}
$$

Based on above, the linear switched system 1 is observable if $O_{n}=\mathfrak{R}^{n}$ [9].

\section{$2.3 \quad$ STABILIZABILITY}

One of the most important features of systems is analyzing their stability. Stabilizability has close relevance with controllability.

Definition 5: (stabilizability) the system 1 for nonzero state $x_{0}$ on switched path $\sigma: R$ a $M$ is stabilizable if there exist an input $u(t)$ such that $\lim _{t \rightarrow \infty} x(t)=0$.
From the above definition, anticipated that the system 1 is stabilizable if all unstable states are controllable.

\section{PREVIOUS WORKS}

Distributed systems present a challenging paradigm for TS problem and considerable literature is devoted to its various aspects. However, the existing techniques generally aim to only "solve" the TS problem and the problem is not approached from a modeling perspective. The scheduling problem has been proven to be NPcomplete except for a few restricted cases[10]. Traditional methods that are used to solve this problem can be generally categorized as either heuristic (list-based scheduling) or random search (such as genetic algorithm, simulated annealing, and particle swarm optimization). A few of the most significant recent directions in this area are highlighted below. Kong, et al. (2008) [11] incorporate Particle Swarm Optimization (PSO), with list scheduling and develop an alternative PSO algorithm for multiprocessor tasks scheduling. Unlike most duplication-based heuristics which try to duplicate all possible ancestor nodes of a given join node, Similarly in (2012)[12], an integrated scheduling model, including two heuristics and a GA-based algorithm for production and distribution operations is introduced that minimizes the required time for all jobs to be completed and delivered to the customer area and the truck returned to the factory.

Yoo (2009) [13] minimizes the total tardiness and total number of processors used in scheduling of soft real-time DAGs on homogeneous multiprocessors systems by a multi-objective genetic algorithm (MoGA). To evaluate chromosomes in a population, Yoo converts multiobjective problem to single objective problem by adaptive weighted approach (AWA). In this approach, the useful information from the current population is used to readjust objective weights and to obtain a search pressure toward a positive ideal point.

In recent years, the study of switched systems has attracted researcher's attention from the area of systems and control [7-9, 14-18]. One of the important related fields is analyzing controllability, observability and controllability of such systems. Ezzine and Haddad [19] considered a class of switched linear systems and gave a criterion for the observability and controllability in one switching period. Then Sun and Zheng in $[4,20]$ gave a necessary and sufficient condition for controllability of linear switched systems and show 
that necessary condition is sufficient condition for a 3 rd order system with two subsystems. Xie and Wang [21] increased their method for controllability on switched systems with time delay. In 2006, Xie and Wang [22] considered the controllability for positive discrete time switched liner systems. In positive discrete time switched systems for any positive initial condition, state remains positive for all time. Ji, et al. [18] investigates the number of switching and design of switching sequences for controllability of switched linear systems and tried to give an acceptable estimation of optimal switching path. Ezzine and Haddad [19] considered observability for switched systems and expanded observability of time variant systems to switched systems. Babaali and Egerstedt [6] studied the case where the switching law is not measurable, and presented some observability conditions. Meng [7] considered singular switched linear systems and gave necessary and sufficient conditions for these systems and conclude in singular systems, observability and controllability are not dual in general. Liberzon [23, 24] shows when all of the subsystems are stable and Commutative the system for any switching path is stable. Wang [16] considered stabilizability for a class of nonlinear systems using Lyapunov criterion and gave a necessary condition for a special class of nonlinear systems. Yang, et al. [14] considered stabilizability for nonlinear systems with unstable modes.

Control theory has been used to design adaptive resource management in different applications such as power management, task scheduling, QoS adaptation in Web servers, and load balancing [25]. In $[26,27]$, the control theory is applied to schedule real-time tasks on distributed systems [25]. Lu, et al. [26] presents a Feedback Control real-time Scheduling (FCS) framework for adaptive real-time systems. They apply a methodology based on control theory to systematically design FCS algorithms to satisfy the transient and steady state performance specifications of real-time systems. In [27], a framework is proposed based on feedback control that incrementally corrects system performance to achieve its target in the absence of initial load and resource assumptions. It allows the designer to specify the desired temporal behavior of system adaptation for a distributed real-time application such as the speed of convergence to desired performance upon load or resource changes. Furthermore, unlike QoS optimization approaches, their solution meets performance guarantees with no accurate knowledge of task execution parameters. This can be considered as a key advantage in a poorly modeled environment.
While the above approaches succeeded in using control theory for task scheduling, their domain of application remained very limited and cover very simplified versions of scheduling, i.e. addressing only QoS in [28] and only one processing unit in [30].

In contrast to the above, we aim to reach a 'general' model of scheduling in state space by using linear switching systems. This modeling space allows the various constraints and applications of scheduling in real environment to be efficiently and faithfully represented, such as those in resource management. The components of the scheduler are developed in such a way that most of the parameters of the problems can be covered. Another important advantage of the proposed method over these similar works is that the modeling of the constraints and also stability of the method is proven using control theory principals; furthermore all of the components of a task scheduling problem are well mapped to corresponding one in control theory. This leads to a powerful theoretical method applicable to practical problems [2].

\section{PROPOSED APPROACH}

This study contributes to task scheduling problem whilst it involves a number of other research areas such as modeling, switching system, distributed systems, control theory and system engineering. These five research areas have great terminologies, thus important words should be redefined at first. In prior research by authors [2, 28], the task scheduling problem is represented as a nonlinear state-space model. Then, this model is linearized and a simple linear switching state-space model of task scheduling problem is introduced [2]. The benefits of linear switching model are that first bypasses all nonlinearity that engendered by constraints of the task scheduling problem and then model allows us simply to utilize all rich control methods for having high efficiency, stable control system. The stability of proposed linear switching model for static task scheduling problem is proved theoretically. Dynamic task scheduling is combined with fuzzy decision making procedure and applied to time-varying heterogeneous multiprocessor systems application in an unstructured environment [28]. Control theory explores all characteristics of the systems from stability through optimality, adaptation through robustness, etc. we have to study Three important subjects about the control model are stabilizability, controllability and Observability. Thus, here we first discuss about 
them theoretically, and then prove some lemma and study them on the proposed linear switching model.

\subsection{PROPOSED STATE SPACE MODEL}

Solving heterogeneous TS problems by traditionnal modeling/search approaches is generally NP complete. Hence, a different modeling paradigm based on nonlinear state space paradigm is proposed here.

Consider a general form of a nonlinear system in discrete time is:

$$
\left.\begin{array}{c}
X[(k+1) \Delta T]=f[X[k \Delta T], U[k \Delta T], k \Delta T] \\
Y[k \Delta T]=g[X[k \Delta T], U[k \Delta T], k \Delta T] \\
k \in\{1,2,3, \ldots\}, \quad X \in \mathbb{R}^{n}, \quad Y \in \mathbb{R}^{n},
\end{array}\right)
$$

Where $f$ and $g$ are nonlinear vector functions, $X$ is a vector of state variables, $U$ is control vector, $Y$ is system output and $\Delta T$ is the length of time steps. Here, we propose to model TS in the above nonlinear form, Equation (4). Various components of this model are shown in subsequent subsections.

\subsubsection{State variables}

State variables in this model correspond to a vector $(X)$ whose size is equal to the number of tasks, and each element has a value between 0 (the task has completed) and 1 (the execution of task has not started). Hence, each element $x_{j}[k]$ corresponds to the remaining process of a given task $j$ at $k^{\text {th }}$ step.

$X[k]=\left[\begin{array}{llllll}x_{1}[k] & x_{2}[k]\end{array} . \quad . \quad x_{T}[k]\right]^{\prime}$

Where $(T=|\boldsymbol{V}|)$ and all of the elements of $X[k]$ are usually initialized to ' 1 '.

\subsubsection{Control Vector}

Control vector $U[k]$ is a matrix $M \times T$, where $T=|\boldsymbol{V}|, M=|\boldsymbol{R}|$, and shows the execution status of each task and each processor in $k^{\text {th }}$ time step. Each element in this matrix is between ' 0 ' and ' 1 ' and depicts available processing power of each processor. The characteristic of this matrix is that at each step there is at most one nonzero element in each row and each column. Element $u_{i, j}$ in $k^{\text {th }}$ time step shows the percentage of utilizing processori in execution of task $j$. If $u_{i, j}[k]=1$, task $j$ is said to be fully executed on resource $i$ in $k^{\text {th }}$ time step. An important constraint in control vector is that all of the tasks, that are simultaneously executed at $k^{\text {th }}$ time step, must be independent from each other. Furthermore, each task can be processed on only one processor, and each processor can process at most one task at any given time.

\subsubsection{Constraints on Controller Design}

These constraints are summarized as follows:

1. $\{$ Initialization $\}$

$$
X[0]=\left[\begin{array}{lllllll}
1 & 1 & . & . & . & 1
\end{array}\right]^{\prime} \quad \text { ) }
$$

2. All of the elements of the control vector $U$ are chosen in range $[0,1]$, i.e.

$$
\forall i, j \quad 0 \leq u_{i, j} \leq 1
$$

3. \{Constraint of Independence\}

$$
\text { if } \exists u_{i, j} \neq 0 \Rightarrow \begin{aligned}
&\left\{\forall j^{\prime} \neq j \quad u_{i, j^{\prime}}\right. \\
&=0 \quad, \quad \\
& \forall i^{\prime} \neq i \quad u_{i^{\prime}, j} \\
&=0\}
\end{aligned}
$$

4. $\{$ Constraint of Predecessors $\}$

$$
\begin{aligned}
& \text { if } \exists u_{i, j} \neq 0 \Rightarrow(\forall r \\
& \in \operatorname{pred}\left(n_{j}\right) x_{r} \\
& =0 \text { ) }
\end{aligned}
$$

5. \{Concurrent Task Independence $\}$

$$
\begin{aligned}
\forall i, j, r, s \quad \text { if } & \left(u_{i, j} \neq 0 \& u_{r, s}\right. \\
& \neq 0) \Rightarrow\left(\mu_{j, s}\right. \\
& \left.=0 \& \mu_{s, j}=0\right)
\end{aligned}
$$

6. $\{$ Constraint of Communication Cost $\}$ If two tasks with precedence relation are scheduled on different processors, non-zero communication cost is considered. Otherwise, communication cost is $\mu_{i, j}=0$.

7. \{Constraint of Task Completion\} After completion of task execution, corresponding processor is freed. In other words,

$$
\begin{aligned}
\forall i, j \quad \text { if } x_{j}[k]= & 0 \\
& \Rightarrow u_{i, j}[k+1] \\
& =0
\end{aligned}
$$

8. \{Constraint of Completeness\}

$$
\forall j \exists i \exists k \quad u_{i, j}[k] \neq 0
$$

\section{- The Non-linear System Function $\boldsymbol{f}$}

The function $f$ in system dynamics, Eq (13) relates the defining parameters of the system such as the vector of state variables $X$, control vector $U$, time step $k$, as well as the DAG. For the TS problem, $f$ is defined in discrete time form as follows, 


$$
\begin{aligned}
X[k+1]=\max & (0, X[k]-\Delta T \\
& \left.* \operatorname{Diag}\left(\left[\frac{1}{\theta_{i j}}\right] * U\right)\right)
\end{aligned}
$$

Where $\Delta T$ is the time step in seconds, Diag is the main diagonal of matrix $A$, and $\theta=\left[\theta_{i j}\right]_{T * M}$ where $\theta_{i j}$ shows computation time of task $i$ on processor $j$. The max operator is chosen to ensure that the state variable does not become negative. Also, the communication cost between tasks (on same or different processors) is shown by $\mu$ which is a $T \times T$ matrix. Communication cost is considered in the process of determining control vectors. These control vectors remain the same when the communication between two tasks is running. Once communication is completed, the processors resume their operation as dictated by the control vector.

\subsection{LINEAR FUNCTION $F_{L}$}

In order to simplify a complex model and to benefit from the extensive theoretical results in the field of linear systems, nonlinear equations are usually converted to linear equations[29]. Linear control systems are much easier to design, however this conversion usually leads to approximation which may be unacceptable. Here, the construct of linear control systems is used to find appropriate linear switching controllers (optimization problem). Nonlinear constraints are considered separately from the linear switching controller; hence the resulting model is exact. By simplifying the model to a linear switching controller, these constraints effectively "reduce" the search space for appropriate controllers[30]. More specifically, we substitute a linear function $f_{L}$ for its nonlinear counterpart $f$ by considering all of the nonlinearities as nonlinear constraints in the controller design process. The resulting model is then converted it to a switched control problem. For this reason, $\max$ and Diag operators should be removed from Equation (13). The following subsections address this process.

\subsection{REMOVING DIAG OPERATOR AND SIMPLIFICATION}

By the following theorem, Diag operator is removed from the product of two general matrices.

Theorem 4- the linear form of $\operatorname{Diag}(Q \times U)$, where $Q$ is a $a \times b$ matrix and $U$ is a $b \times a$ matrix, is $\operatorname{Diag}(Q \times U)$

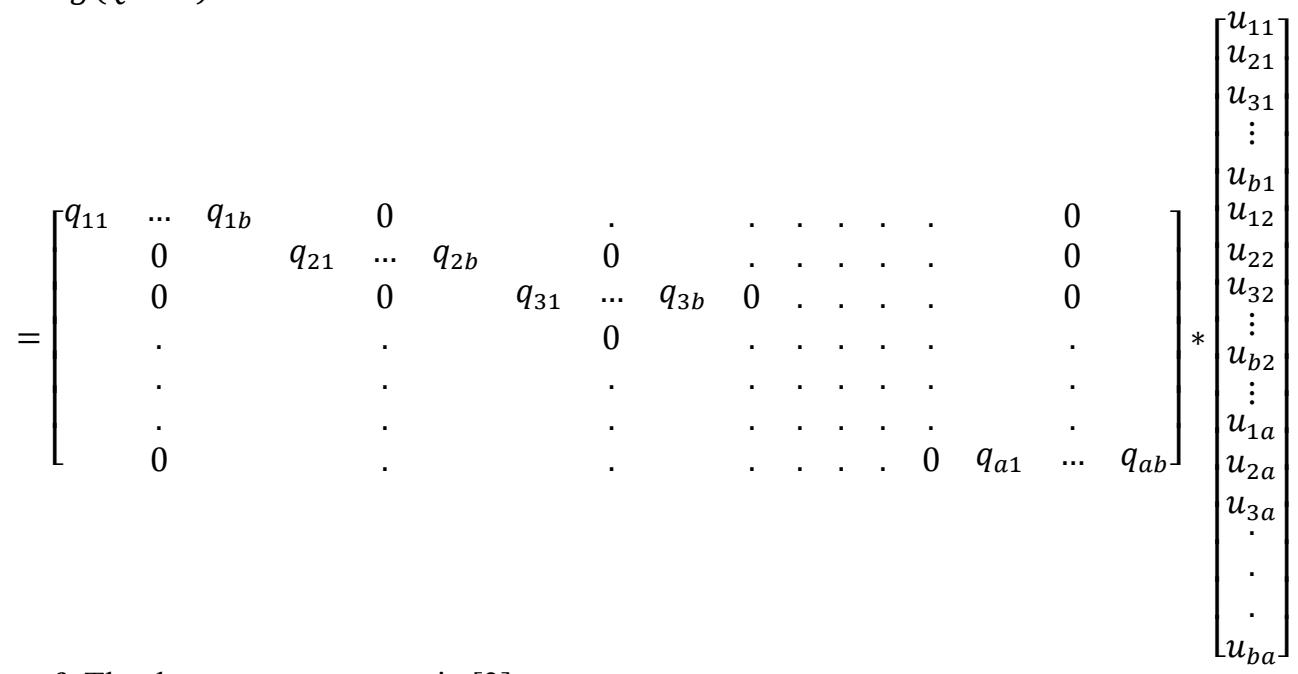

Proof- The theorem was proven in [2].

Based on Theorem 4, Equation (13) is converted to 


$$
X[k+1]=\max \left(0, X[k]-\Delta T *\left[\begin{array}{ccccccccc}
\bar{q}^{1} & 0 & . & . & . & . & . & . & 0 \\
0 & \bar{q}^{2} & 0 & . & . & . & . & . & 0 \\
0 & 0 & \bar{q}^{3} & 0 & . & . & . & . & 0 \\
. & . & . & . & . & . & . & . & 0 \\
. & . & . & . & . & . & . & . & . \\
. & . & . & . & . & . & . & . & . \\
0 & . & . & . & . & . & . & . & . \\
0 & . & . & . & . & . & 0 & \bar{q}^{T}
\end{array}\right] *\left[\begin{array}{c}
\bar{u}^{1} \\
\bar{u}^{2} \\
\cdot \\
. \\
\cdot \\
\bar{u}^{T}
\end{array}\right]\right)
$$

Where $Q_{T \times M}=\frac{1}{\theta_{T \times M}}$.

\subsubsection{LINEARIZATION OF MAX OPERATOR}

Here, we remove $\max$ operator by considering a constraint on designing control vectors.

In other words, we zero out the corresponding tasks in control vector in an ascending order of execution times. By this method, if execution of one task has ended, its corresponding processor is freed, and its corresponding elements of state variables do not become negative.

By considering this new constraint on control vector, we reach a linear model as follows:

Where $Q_{T \times M}=\frac{1}{\theta_{T \times M}}$

$$
X[k+1]=X[k]-\Delta T *\left[\begin{array}{ccccccccc}
\bar{q}^{1} & 0 & . & . & . & . & . & . & 0 \\
0 & \bar{q}^{2} & 0 & . & . & . & . & . & 0 \\
0 & 0 & \bar{q}^{3} & 0 & . & . & . & . & 0 \\
. & . & . & . & . & . & . & . & 0 \\
. & . & . & . & . & . & . & . & . \\
. & . & . & . & . & . & . & . & . \\
0 & . & . & . & . & . & . & . & . \\
0 & . & . & . & . & . & . & 0 & \bar{q}^{T}
\end{array}\right] *\left[\begin{array}{c}
\bar{u}^{1} \\
\bar{u}^{2} \\
. \\
. \\
. \\
\bar{u}^{T}
\end{array}\right]
$$

Theorem 8- the linear form of $\operatorname{Diag}(Q \times U)$, where $Q$ is a $a \times b$ matrix and $U$ is a $b \times a$ matrix, is

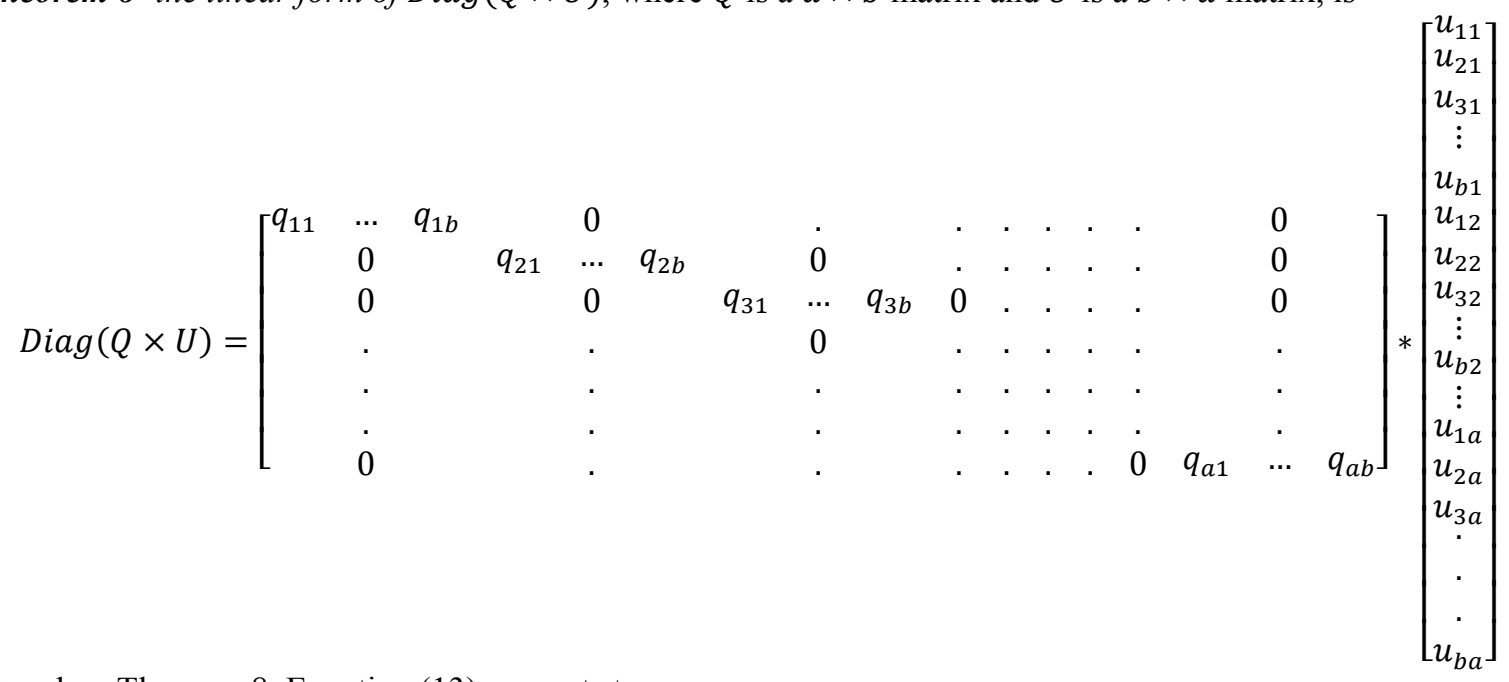

Based on Theorem 8, Equation (13) converts to

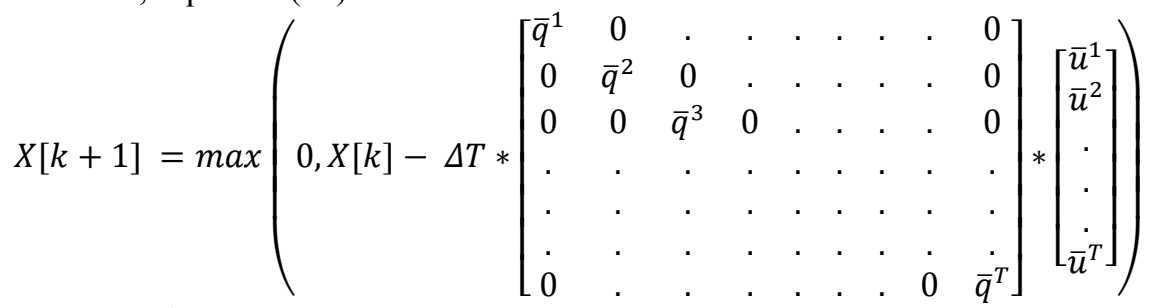

Where $Q_{T \times M}=\frac{1}{\theta_{T \times M}}$. 
Furthermore, it will be studied in controllability section that in this model as $A=I$ therefore $W_{1}=W_{2}=\mathrm{K}=W_{n}$ and thus only checking the rank of matrix $W_{1}$ simultaneously satisfies the necessary and sufficient conditions.

In this model $W_{1}=B_{1}+B_{2}+\mathrm{K}+B_{N}=Q^{\prime}$ is chosen so that it has complete rank. Therefore this model is state complete controllable. Considering the axioms on the passage and as a result, this system is also stabilizable.

\section{STABILITY, ROBUSTNESS, CONTROLLABILITY AND OBSERVABILITY OF THE PROPOSED APPROACH}

Some more important topics of the control theory are controllability, observability and stabilizability; in this section these topics will be discussed. In this paper, for simplicity we assume the system 1 is revisable, in the other word $A_{i}$ is nonsingular.

\section{$5.1 \quad$ STABILITY ANALYSIS}

In control viewpoint, it is necessary to prove some basic theories such as stability that illustrates the transition and final response for system [29-31]. Extending this definition to the task scheduling paradigm enables the theoretical study of such features. In other words, with the present approach, it is now possible to rigorously study "convergence" behavior of a scheduling approach. The set of $\mathrm{C}$ controllers that stabilize the nominal feedback system $\left(\mathrm{C}, P_{0}\right)$ are described by below the Yula Parameterization theorem as follow:

Theorem 5 [32]- let $P_{0}$ be an open loop stable plant. Then the set of all controllers stabilizing the nominal feedback system is:

$$
C=\left\{C(s)=\frac{Q(s)}{1-P_{0(s)} Q(s)}\right\}
$$

Where $\mathrm{Q}(\mathrm{s})$ is proper and stable. Yula also introduces a similar theorem for MIMO systems. In following the above work, we introduce here the below theorem to address the convergence and stability of TS problems.

Theorem 6 - If control vectors $U^{1}, U^{2}, \ldots, U^{k}$ satisfy all constraints as mentioned in Section 4.1, at step $a_{k}$ we have

$$
\begin{aligned}
X\left[a_{k}\right]=\max ( & 0, X\left[a_{k-1}\right] \\
& \left.-\operatorname{Diag}\left(\left[\frac{1}{\theta}\right] * U^{k}\right)\right)
\end{aligned}
$$

Theorem 7 (Stability) - If control vectors $U^{1}, U^{2}, \ldots, U^{k}$ satisfy all constraints as mentioned in Section 4.1, we achieve $\left\|X\left[a_{k}\right]\right\|=0$, at step $a_{k}$.

\subsubsection{EXTENDED MODEL OF TASK SCHEDULING IN STATE SPACE [2]}

Here, we introduce an extended model, based on our earlier model [2] on static task scheduling, to consider time varying changes in the environment as follows:

$$
\begin{aligned}
X[k+1]=\max (0, X[k]-\Delta T \\
\\
* \operatorname{Diag}\left(\frac{1}{\left(\sum_{j} C L\left(\lambda_{i}^{j}\right)\right) * \frac{1}{\xi}}\right] \\
* U))
\end{aligned}
$$

$\operatorname{Diag}(A)$ is the main diagonal of matrix $A$, and $C L\left(\lambda_{i}^{j}\right)$ stands for the number of clocks that a processor should spend for instruction $\lambda_{i}^{j}$ to be completed.

With Equation (21), only clock requirements of instructions in task $\mathrm{i}$ and speed of processors are required. In comparison with our earlier model [2] on static task scheduling, the required time for completing any task on any processor is not known as a priori.

One of the important factors in control systems is robustness. Using robust control methods, the stability and performance of control system is guaranteed in the presence of uncertain parameters and disturbances. In the next theorems, the robustness of the proposed approach is considered to demonstrate that it is applicable in uncertain environment [28].

\section{Theorem 9 (Boundedness)-}

$\forall D \in\{s \square t$ of all DAGs with finite height $H\} \Rightarrow$ $\psi(D)<H(M+Z)<\infty$. In other words, makespan $\psi$ is bounded for all finite $D A G s$ with arbitrary weights of nodes and edges and is a linear multiple of task height $H$ considering the following two conditions: 
H. Tabatabaee et al. / International Journal of Computer Networks and Communications Security, 1 (4), September 2013

1. The execution time of each task on any arbitrary processor is bounded.

2. The communication time between any two arbitrary processors is bounded.

Proof- Consider a DAG that is divided according to its levels of nodes. By definition, it is assumed that the nodes at same level are independent of each other and can run in parallel. Furthermore, it is assumed that all of the edges between two levels in a DAG have the ability to communicate simultaneously. Therefore, all of the tasks at same level $i$, can be executed in $t s_{i}$ units:

$$
\begin{gathered}
t s_{i}=\operatorname{Max}\left\{E T_{i j}\right\}, i=0,1,2,3, \ldots, H \\
j=1,2, \ldots, \beta \\
\psi \leq \sum_{i=0}^{H-1} c_{i}+\sum_{i=0}^{H} t s_{i}=t s_{0}+c_{0}+t s_{1}+c_{1}+\cdots+t s_{H-1}+c_{H-1}+t s_{H}
\end{gathered}
$$

Where $H$ is the height of DAG. Below, we will show the above sum to be bounded.

Assuming the height of any finite DAG, $H$, to be bounded, i.e.

$\exists H_{\max }<\infty, H \leq H_{\max }$

And also because execution time of each task is bounded, $t s_{i}$ is also bounded, i.e.,

$t s_{i} \leq M, \quad$ for $M=\max \left\{M_{i}\right\}, \quad i=0,1,2, \ldots, H$

$M_{i}$ is the maximum execution time of the tasks at $i^{t h}$

level.

$$
\begin{aligned}
& \psi \leq t s_{0}+c_{0}+t s_{1}+c_{1}+\cdots+t s_{H-1}+c_{H-1}+t s_{H} \\
& \Rightarrow \psi \leq M_{0}+B_{0}+M_{1}+B_{1}+\cdots+M_{H-1}+B_{H-1}+M_{H} \\
& \Rightarrow \psi \leq H \times M+(H-1) \times Z \\
& \Rightarrow \psi \leq H \times M+(A-1) \times Z \\
& \Rightarrow \psi \leq H * M+H * Z=H(M+Z) .
\end{aligned}
$$

Hence,

the summation of finite bounded numbers is a bounded number,

$\left.\begin{array}{c}\exists W<\infty, M+Z<W \\ \quad \exists X<\infty, H<X\end{array}\right\} \Rightarrow H(M+Z)<W X$.

Finally, since the multiplication of finite bounded numbers is a bounded number,

$\exists \eta<\infty, W X<\eta \Rightarrow \psi<\eta$.

And theorem is proofed.

\subsection{ROBUSTNESS ANALYSIS}

Proposed model has capability of facing with uncertainty in environment. In a real world task scheduling problem processing power of resources has uncertainty. In proposed model, resources can perform another task, simultaneously; therefore, it is not sufficient know resource's processing speed and it must be considered uncertainty in resource's processing speed. Processing power of resources determines execution time of tasks; therefore, uncertainty shows itself in $q$ matrix in formula (17) and $q$ is converted to $q+\Delta q$; therefore this formulation is converted to: 
H. Tabatabaee et al. / International Journal of Computer Networks and Communications Security, 1 (4), September 2013

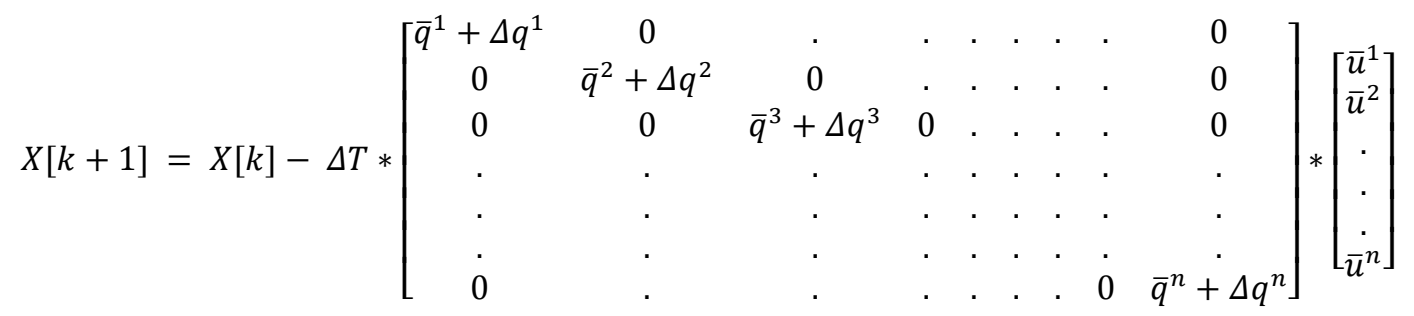

And then,

$$
\begin{aligned}
& X[k+1]=X[k]-\Delta T *\left[\begin{array}{ccccccccc}
\bar{q}^{1} & 0 & . & . & . & . & . & . & 0 \\
0 & \bar{q}^{2} & 0 & . & . & . & . & . & 0 \\
0 & 0 & \bar{q}^{3} & 0 & . & . & . & . & . \\
. & . & . & . & . & . & . & . & 0 \\
. & . & . & . & . & . & . & . & . \\
. & . & . & . & . & . & . & . & . \\
0 & . & . & . & . & . & . & . & . \\
0 & . & . & . & . & 0 & \bar{q}^{n}
\end{array}\right] *\left[\begin{array}{c}
\bar{u}^{1} \\
\bar{u}^{2} \\
. \\
. \\
. \\
\bar{u}^{n}
\end{array}\right]-\Delta T
\end{aligned}
$$

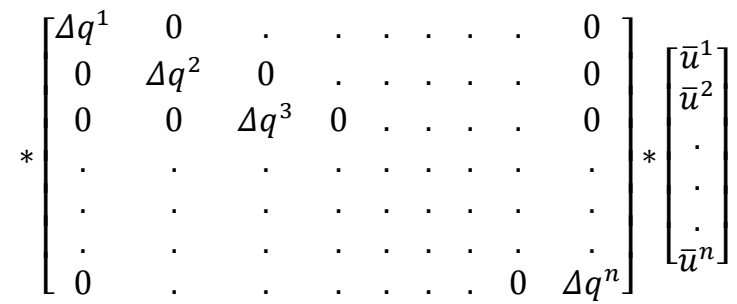

And

$$
X(k+1)=A X(k)+F_{i}+\Delta Q
$$

Based on formulation (32) proposed model has capability of facing with uncertainty in processing power of resources.

Also if there is uncertainty in speed of links in network or even in its topology, our model can handle it. Since we consider communication delays as a constraint it is possible to face it as follows:

Successor $\left(\boldsymbol{T a s k}_{\boldsymbol{i}}\right) \triangleq$ Set of successor $\boldsymbol{T a s k}_{\boldsymbol{i}}$ Resource $\left(\boldsymbol{T a s k}_{\boldsymbol{i}}\right)$

$\triangleq$ Resource number Allocated to Task $_{\boldsymbol{i}}$

$R E T(T)$ stands for run-time execution time of task $T$, $E T(T)$ is the execution time of task $T$, and $\Delta e(\Delta e \in$ $\mathbb{R})$.is change in execution time due to disturbance. This means that if execution time of each task $T$ sum Theorem 10-2 (Robustness in presence of the uncertainty on communication time of between tasks) -

$c\left(t_{i}, t_{j}\right)$ Represents the communication time between taskst $t_{i}$ and $t_{j}$ and $R c\left(t_{i}, t_{j}\right)$ represents the run-time communication time between er and then this theorem is proven.

\section{Communication Time $\left(\boldsymbol{T a s k}_{\boldsymbol{i}}\right)$}

$$
=\left\{\begin{array}{cr}
\mathbf{0} & \text { Successor }^{\text {Task } \left._{\boldsymbol{i}}\right)} \\
\mathbf{0} & \text { Resource }\left(\boldsymbol{T a s k}_{\boldsymbol{i}}\right)=\text { Resol } \\
\underset{\mathrm{m} \in \operatorname{Successor}\left(\boldsymbol{T a s k}_{\boldsymbol{i}}\right)}{\max \left\{\mu_{\mathrm{ij}}+\Delta \mu\right\}} & \text { Otherwise }
\end{array}\right.
$$

Total Communication Time

$=\sum_{i=1}^{n}$ Communication Time $\left(\boldsymbol{T a s k}_{\boldsymbol{i}}\right)$

Theorem 10-1 (Robustness in presence of the uncertainty on execution time of tasks) -

$\forall D \in D A G s \forall T \in \operatorname{Tasks}(D) \ni \Delta e \in \mathbb{R}, \operatorname{RET}(T)<$ $\infty, \operatorname{RET}(T)=E T(T)+\Delta E \Rightarrow \psi(D)<\beta<\infty$.

with that is bounded, makespan $\psi$ is also bounded.Proof-Since $\Delta e$ is bounded, $t s_{i}+\Delta e$ is also bounded. By application of Theorem 1, then this theorem is proven.

$$
\begin{aligned}
& \forall D \in D A G s \forall t_{i}, t_{j} \in D \quad \ni \Delta e \in \mathbb{R}, c\left(t_{i}, t_{j}\right) \neq 0, \\
& R c\left(t_{i}, t_{j}\right)<\infty, R c\left(t_{i}, t_{j}\right)= \\
& c\left(t_{i}, t_{j}\right)+\Delta E \Rightarrow \psi(D)<\beta<\infty . \\
& \text { taskst }_{i} \text { and } t_{j} \text { and } \Delta e(\Delta e \in \mathbb{R}) \text { is change in }
\end{aligned}
$$
communication time due to disturbance.

Proof-Since $\Delta e$ is bounded, $C_{i}+\Delta e$ is a bounded numb 


\subsection{CONTROLLABILITY ANALYSIS}

Lets consider the proposed linear switched model, because the state-space matrix $\mathrm{A}=\mathrm{I}$, thus the controllability axioms are simplified to checking of a matrix rank. The simplified is done as:

$$
\begin{gathered}
\left\langle A_{i} \mid B_{i}\right\rangle=B_{i}+A_{i} B_{i}+\mathrm{K}+A_{i}^{n-1} B_{i}: \\
W_{1}=\sum_{i=1}^{N}\left\langle A_{i} \mid B_{i}\right\rangle=B_{1}+B_{2}+\mathrm{K}+B_{i} \\
W_{2}=\sum_{i=1}^{N}\left\langle A_{i} \mid W_{1}\right\rangle=B_{1}+B_{2}+\mathrm{K}+B
\end{gathered}
$$

M

$$
W_{n}=\sum_{i=1}^{N}\left\langle A_{i} \mid W_{n-1}\right\rangle=B_{1}+B_{2}+\mathrm{K}+
$$

As it is realized, $W_{1}=W_{2}=\mathrm{K}=W_{n}$ thus studies on $W_{i}, i=2, \ldots, n$ have no any new result and checking the rank of matrix $W_{1}$ is adequate. As mentioned previously, the necessary condition of controllability of linear switching system is $W_{n}=\Re^{n}$, also the sufficient condition is $W_{1}=\mathfrak{R}^{n}$ and here is seen that in this model $W_{n}=W_{1}$ so $W_{1}=\mathfrak{R}^{n}$ is simultaneously satisfies the necessary and sufficient conditions. $W_{1}=\Re^{n}$ Means the rank of matrix $W_{1}$ is equal to $n$. As a result, the simplified controllability axiom for the proposed model is expressed:

Lemma 1:

The proposed linear switching model is controllable if and only if the rank of matrix $W_{1}$ (composed of vectors $B_{i}$ ) is equal to $n$.

In the proposed method, the matrix $W_{1}=B_{1}+B_{2}+\mathrm{K}+B_{N}=Q^{\prime}$ is chosen so that it has complete rank, thus the introduced linear switched system is completely controllable.

\subsection{OBSERVABILITY ANALYSIS}

As expressed at controllability, in this model the matrix $A=I$ therefore we can also simplified the observibillity axioms.

$$
\begin{aligned}
& \left\langle A_{i}^{T} \mid C_{i}^{T}\right\rangle=C_{i}^{T}+A_{i}^{T} C_{i}^{T}+\mathrm{K}+\left(A_{i}^{n}\right)^{T} C_{i}^{T} \\
& O_{1}=\sum_{i=1}^{N}\left\langle A_{i}^{T} \mid C_{i}^{T}\right\rangle=C_{1}^{T}+C_{2}^{T}+\mathrm{K}+C_{n}^{T} \\
& O_{2}=\sum_{i=1}^{N}\left\langle A_{i}^{T} \mid O_{1}\right\rangle=C_{1}^{T}+C_{2}^{T}+\mathrm{K}+C_{n}^{T} \\
& \mathrm{M} \\
& O_{n}=\sum_{i=1}^{N}\left\langle A_{i}^{T} \mid O_{2}\right\rangle=C_{1}^{T}+C_{2}^{T}+\mathrm{K}+C_{n}^{T}
\end{aligned}
$$

At the formula above, as $O_{1}=O_{2}=\mathrm{K}=O_{n}$ so study on $O_{i}, i=2, \ldots, n$ has no new results and only checking the rank of $O_{1}$ is enough. As the result, the observability axiom is simplified as bellow:

\section{Lemma 2:}

This model is observable if and only if the rank of matrix $O_{1}$ that comprised of the vectors $C_{i}^{T}$ is equal to $n$, and the matrix $O_{1}=C_{1}^{T}+C_{2}^{T}+\mathrm{K}+C_{n}^{T}=I$ is chosen so that is has complete rank, therefore, the linear switched system is observable.

\section{CONCLUSION}

Control theory has some important concepts; here, we study on the controllability, observability and stabilizability of the model we introduced before as linear switched model of task scheduling problem. The controllability, observability and stabilizability is theoretically studied and proved for the proposed model. Considering the characteristics of the model, in this paper, we simplify these conditions and bring up some lemma. Also, we analytically prove that this model is controllable, observable and stabilizable. The major contributions of this work includes: first, modeling the task scheduling problem by linear switching state space equations that simplify the controller (scheduler) design process; second, proposing a general modeling framework, not just an algorithm, to solve the scheduling problem. The model can be adapted to other variations of the scheduling problem; third, one is that the model introduces various theoretical measures of performance, such as system stability for the problem of task scheduling. Finally, the proposed model is capable of supporting multiple constraints in a real environment such as considering the small 
time steps as well as the optimal routing between processing nodes.

\section{REFERENCES}

[1] A. J. Page and T. J. Naughton, "Dynamic task scheduling using genetic algorithms for heterogeneous distributed computing," in proceedings of the 19th IEEE International Parallel and Distributed Processing Symposium (IPDPS'05) USA, 2005, pp. 152159.

[2] H. Tabatabaee and M.-R.Akbarzadeh-T., "The Linear Switching State Space: A New Modeling Paradigm for Task Scheduling Problems," in International Journal of Innovative Computing, Information and Control Volume 9, Number 4, April (2013) ISSN 1349-4198, pp. 1651-1677.

[3] C. Daizhan, "Global Controllability of Switched Nonlinear Systems," in Decision and Control, 2006 45th IEEE Conference on, 2006, pp. 3742-3747.

[4] Z. Sun and D. Zheng, "On reachability and stabilization of switched linear systems," Automatic Control, IEEE Transactions on, vol. 46, pp. 291-295, 2001.

[5] G. Xie, D. Zheng, and L. Wang, "Controllability of switched linear systems," Automatic Control, IEEE Transactions on, vol. 47, pp. 1401-1405, 2002.

[6] M. Babaali and M. Egerstedt, "Observability of switched linear systems." vol. 2993, 2004, pp. 48-63.

[7] B. Meng, "Observability Conditions of Switched Linear Singular Systems," in Control Conference, 2006. CCC 2006. Chinese, 2006, pp. 1032-1037.

[8] G. Xie and L. Wang, "Necessary and sufficient conditions for controllability and observability of switched impulsive control systems," Automatic Control, IEEE Transactions on, vol. 49, pp. 960-966, 2004.

[9] Z. Sun and S. S. Ge, "Analysis and synthesis of switched linear control systems," Automatica, vol. 41, pp. 181-195, 2005.

[10]M. Garey and D. Johnson, Computers and Intractability: A Guide to the Theory of NPCompleteness, 1 ed.: W. H. Freeman, 1979.

[11]X. Kong, J. Sun, and W. Xu, "Permutationbased Particle Swarm Algorithm for Tasks Scheduling in Heterogeneous systems with Communication Delays," Computational Intelligence Research, vol. 4, pp. 61-70, 2008.

[12]C.-H. Liu, B.-Y. Leu, and S.-Y. Hsu, "SCHEDULING OF PARALLEL
MACHINES WITH JOB DELIVERY COORDINATION," International Journal of Innovative Computing, Information and Control, vol. 8, pp. 553-566, 2012.

[13]M. Yoo, "Real-time task scheduling by multiobjective genetic algorithm," Systems and Software, vol. 82, pp. 619-628, 2009.

[14]H. Yang, B. Jiang, V. Cocquempot, and H. Zhang, "Stabilization of Switched Nonlinear Systems With All Unstable Modes: Application to Multi-Agent Systems," Automatic Control, IEEE Transactions on, vol. 56, pp. 2230-2235, 2011.

[15]C. Shen, J. Haifeng, and H. Yanyan, "On stabilization conditions of linear stochastic switched systems," in Intelligent Control and Information Processing (ICICIP), 2011 2nd International Conference on, 2011, pp. 19-21.

[16] W. Min, F. Jiaxin, G. M. Dimirovski, and Z. Jun, "Stabilization of switched nonlinear systems using multiple Lyapunov function method," in American Control Conference, 2009. ACC '09., 2009, pp. 1778-1782.

[17]C. Meiyan, M. Xiaowu, and F. Jianyin, "Stabilization of discrete-time switched systems," in Intelligent Control and Automation, 2008. WCICA 2008. 7th World Congress on, 2008, pp. 4100-4102.

[18]K. Shin, M. Cha, M. Jang, J. Jung, W. Yoon, and S. Choi, "Task scheduling algorithm using minimized duplications in homogeneous systems," Parallel and Distributed Computing, vol. 68, pp. 1146-1156, 2008.

[19] J. Ezzine and A. H. Haddad, "On the Controllability and Observability of Hybrid Systems," in American Control Conference, 1988, 1988, pp. 41-46.

[20]Z. Sun, S. S. Ge, and T. H. Lee, "Controllability and reachability criteria for switched linear systems," Automatica, vol. 38, pp. 775-786, 2002.

[21]G. Xie and L. Wang, "Controllability and stabilizability of switched linear-systems," Systems and Control Letters, vol. 48, pp. 135155, 2003.

[22] G. Xie and L. Wang, "On Reachability and Controllability of Positive Discrete-time Switched Linear Systems," in Systems, Man and Cybernetics, 2006. SMC '06. IEEE International Conference on, 2006, pp. 45754579.

[23]D. Liberzon and A. S. Morse, "Basic problems in stability and design of switched systems," Control Systems, IEEE, vol. 19, pp. 59-70, 1999. 
H. Tabatabaee et al. / International Journal of Computer Networks and Communications Security, 1 (4), September 2013

[24]D. Liberzon, J. P. Hespanha, and A. S. Morse, "Stability of switched systems: A Lie-algebraic condition," Systems and Control Letters, vol. 37, pp. 117-122, 1999.

[25]D. C. Marinescu, "Application of Control Theory to Task Scheduling on a Cloud," in Cloud Computing and Computer Clouds Orlando, FL 32816, USA: University of Central Florida, 2012.

[26] C. Lu, J. A. Stankovic, G. Tao, and S. H. Son, "Feedback Control Real-Time Scheduling: Framework, Modeling, and Algorithms," Special issue of Real-Time Systems Journal on Control-Theoretic Approaches to Real-Time Computing, vol. 23, pp. 85-126, 2002.

[27] J. Stankovic, T. He, T. Abdelzaher, M. Marley, G. Tao, S. Son, and C. Lu, "Feedback Control Scheduling in Distributed Real-Time Systems," in 22nd IEEE Real-Time Systems Symposium, Charlottesville, VA, USA, 2001, pp. 59 - 70.

[28]H. Tabatabaee, M.-R.Akbarzadeh-T., and N. Pariz, "Linear Switching State Space (LS3) for Fuzzy Dynamic Task Scheduling in Unstructured Heterogeneous Multiprocessor Systems," Submitted to Journal of Zhejiang University Science C (Computers \& Electronics)

[29] K. Ogata, Discrete-Time Control Systems, 2 ed.: Prentice Hall, 1995.

[30]Z. Sun, Switched Linear Systems: Control and Design (Communications and Control Engineering) [Hardcover]: Springer, 2005.

[31] C.-T. Chen, Linear System Theory and Design, 2 ed.: Oxford University Press, USA, 1995.

[32] H. Ozbay, Introduction to feedback control theory. 